Research Article

\title{
Effect of Football on Fatigue of Patients with Breast Cancer Treated with Nano-Chemotherapy
}

\author{
Yu Zhang $(\mathbb{D}$, Haonan Niu, and Zhiwei Peng \\ Hunan Normal University, Changsha 410006, Hunan, China \\ Correspondence should be addressed to Yu Zhang; zhangyu1978@hunnu.edu.cn
}

Received 7 August 2020; Revised 9 September 2020; Accepted 16 September 2020; Published 5 October 2020

Academic Editor: Tifeng Jiao

Copyright (c) $2020 \mathrm{Yu}$ Zhang et al. This is an open access article distributed under the Creative Commons Attribution License, which permits unrestricted use, distribution, and reproduction in any medium, provided the original work is properly cited.

\begin{abstract}
Cancer-related fatigue is one of the most common and uncontrollable subjective and persistent fatigue feelings in patients after breast cancer surgery, which seriously affects the rehabilitation effect and quality of life of patients. The purpose of this study was to investigate the effect of football intervention on cancer-related fatigue and quality of life in patients with breast cancer after nanochemotherapy. The objective of this study is to explore the exercise program that can make patients actively carry out rehabilitation exercise and achieve good rehabilitation effect, so as to provide a theoretical and empirical basis for the study of cancer fatigue and quality of life of patients after breast cancer surgery. In this study, a quasi-experimental study method was used to conveniently select 60 breast cancer outpatients with tumor-related fatigue symptoms in a tertiary hospital in Liaoning Province. According to the convenience group, they were divided into a control group and an intervention group, 30 cases in each group. The control group received routine hospital nursing, while the intervention group received football intervention for 6 months on the basis of routine hospital nursing. The intervention measures include explaining the related knowledge of cancer-related fatigue, helping patients develop football projects, guiding patients to record football sports diaries, checking patients' football sports diary records at the beginning of each chemotherapy cycle, and discussing football sports feelings with patients. After six months of intervention, the researchers assessed cancer-related fatigue symptoms and quality of life. Football can improve the fatigue, physiological, psychological, and psychological states of breast cancer patients after nano-chemotherapy; reduce fatigue, anxiety, and depression; improve sleep; and ultimately improve the quality of life.
\end{abstract}

\section{Introduction}

Breast cancer [1] is gradually becoming the first malignant tumor threatening women's health. The incidence rate of breast cancer worldwide is $15 \%$ of all cancers, accounting for $37 \%$ of all cancer in women, accounting for $23 \%$ of all cancer deaths in women. Globally, the higher the geographic dimension, the higher the incidence rate; the high incidence areas are North America and Northern Europe; the central region is Israel, South America, and southern Europe; the low incidence areas are Asia and Africa. The incidence rate of incidence of white women in the United States is high. About 1 of the 8-9 people may have breast cancer, and the incidence rate is about $13.7 \%$. The incidence rate of Asian women is $4-7 \%$.

In order to explore the effect of this intervention method, football exercise intervention was carried out for 6 months for breast cancer outpatients with cancer-related fatigue [2] symptoms. According to the principle of randomized controlled grouping, the patients were divided into an intervention group and a control group. Cancer-related fatigue scale (CFS), quality of life scale (WHOQOL-BREF), Pittsburgh Sleep Scale [3] (PSQI), and anxiety and depression scale [4] (SAS, SDS) were used for investigation and evaluation. Cancer-related fatigue, quality of life, sleep quality, anxiety, and depression of the two groups were regularly analyzed.

Football intervention can effectively reduce the fatigue degree of patients with breast cancer, and the rehabilitation effect is better than the traditional rehabilitation exercise after breast cancer surgery. Football intervention can improve the sleep quality of patients, reduce the anxiety and depression of patients, have good exercise compliance, and effectively improve the quality of life of patients with rehabilitation, and the effect is better than the control group of 
traditional rehabilitation exercise. Exercise is a safe and feasible intervention method, which can effectively prevent the deterioration of cancer-related fatigue symptoms of patients with nano-chemical chemotherapy technology, but the effect of quality of life needs further research.

In this paper, the cancer-related fatigue status and its influencing factors of patients with breast cancer treated by nano-chemical chemotherapy [5] after breast cancer surgery were discussed. Only when we have a certain understanding of the factors can we design a reasonable experiment. Because of the various causes of cancer-related fatigue, we mainly discuss it by common factors. Using the design of a clinical randomized controlled trial, 60 patients were selected as volunteers in a hospital in Liaoning Province, and they were divided into two groups, one for the intervention group and the other for the control group. Finally, we concluded that football can reduce the fatigue, anxiety, and depression of patients with breast cancer after nano-chemotherapy technology, improve the sleep of patients, and ultimately improve the quality of life of patients.

\section{Cancer-Related Fatigue and Its Influencing Factors in Patients with Breast Cancer after Chemotherapy}

2.1. Cancer-Related Fatigue in Breast Cancer Patients Undergoing Chemotherapy. Tumor-related fatigue is the most common and destructive clinical symptom in breast cancer patients undergoing chemotherapy. Relevant studies have shown that the incidence of cancer-related fatigue in breast cancer patients with chemotherapy is as high as $97 \%$. In this study, breast cancer patients with chemotherapy have more serious physical fatigue, and emotional fatigue and cognitive fatigue are less. In this study, cancer-related fatigue is divided into two categories: peripheral fatigue [6] and central fatigue [7].

Peripheral fatigue includes somatic fatigue [8]. Peripheral fatigue is related to the activation of the vagal afferent nerve and changes in muscle metabolism. The tumor and tumor treatment can stimulate the peripheral nerve to release neuroactive substances and activate the vagal afferent nerve, which can inhibit the activity of skeletal muscle. As a result, the reduction of skeletal muscle activity leads to body fatigue and body fatigue.

Central fatigue, including emotional fatigue and cognitive fatigue, is mainly related to the imbalance of various nerve bundles and substances in the brain. In this study, physical fatigue is more serious than emotional fatigue and cognitive fatigue which may be due to changes in muscle metabolism, resulting in abnormal sarcoplasmic endoplasmic reticulum function [9], which increases intracellular calcium levels and decreases mitochondrial adenosine triphosphate [10] (ATP) synthesis. In addition, some patients may suffer from anemia and insufficient nutrition intake, which leads to the reduction of ATP synthesis nutrients and the change of ATP metabolism in muscle cells [11].

The influence of cancer-related fatigue on the physiological dimensions of breast cancer patients after chemotherapy is as follows: easy to fatigue, want to lie down and rest, the body becoming heavy, feeling tired, emotional irritability, and not knowing how to deal with fatigue. The effects of chemotherapy on the emotional dimension of breast cancer patients include lack of vitality, loss of interest in many things, and inability to concentrate and motivate themselves to do things. The effect of chemotherapy on the cognitive dimension of breast cancer patients is that patients become careless, more likely to say wrong words, become very forgetful, and have a slow reaction.

\subsection{Common Factors of Cancer-Related Fatigue in Patients} with Breast Cancer after Chemotherapy. In this study, we learned from the literature that the demographic data of cancer-related fatigue in breast cancer chemotherapy patients include different numbers of children, different personality types, and different degrees of housework and appetite. The results show that the more the children, the higher the score of fatigue; the more the patient is extroverted, the less the fatigue symptoms; the more the housework, the heavier the degree of fatigue; the better the appetite, the lighter the degree of fatigue. The reason is the fact that during hospitalization, children take turns to take care of patients in the hospital except for work. As mothers, they feel sorry for their children when they see them working hard every day. On the contrary, they are more worried about their health. They have an increased degree of distraction, heart weakness, and physical insufficiency. At the same time, for patients in the process of cancer treatment, the use of chemotherapy drug tolerance is different; there are some side effects; at this time, physiological and psychological states have a certain impact. Personality is to take an optimistic and positive attitude toward extroverted patients, believe in their own ability, actively collect and understand the relevant knowledge of breast cancer treatment, seek help from others, and correctly understand and actively deal with the disease, so it has a positive impact on psychology and is full of confidence in cancer treatment. Introverted patients do not like to communicate with others. They are quiet and conservative in their lives. They do not want to reveal their ideas to others. They are reluctant to talk to others when they are faced with problems related to the disease. They tend to focus on themselves and their own events. They closed themselves and did not seek help from others and society. This is consistent with many studies. When patients return to their families, they habitually return to their previous roles in life, taking care of their families and doing housework. However, their physical condition and mood have been greatly different from those before the disease, and the degree of fatigue has also increased. Multivariate analysis showed that the frequency of housework had a significant impact on cancer-related fatigue. The higher the frequency of housework, the more serious the degree of fatigue. In the process of chemotherapy, the most common side effect of chemotherapy is a gastrointestinal reaction, which can lead to loss of appetite, nausea, and vomiting and reduce energy intake. In the process of treatment, some patients' pain and insomnia exacerbated the body's energy consumption. When the body's energy supply is not enough to maintain 
the body's energy consumption, fatigue symptoms will appear in the body. Therefore, in the process of talking with cancer patients, clinical medical staff should pay more attention to observe the emotions of patients and find the root causes of such emotions, so as to better communicate with patients and their families and achieve good adjuvant treatment effect [12].

\subsection{Relationship between Anxiety, Depression, and Cancer-} Related Fatigue. The results showed that out of 60 cases of breast cancer chemotherapy patients, $83 \%$ have an anxiety state, and $77 \%$ have a depression positive rate. The reason for this result may be that the secondary sexual characteristics of women after breast cancer surgery have changed, affecting the family and social life of patients. Patients who have body image defects and who are unable to adapt to the role changes will produce a sense of inferiority and helplessness and will have increased depression. For women, it is more likely to cause depression than traditional mastectomy. Due to the different chemotherapy time and drug concentration, breast cancer patients are often accompanied by some adverse reactions, which limit the activity of patients and affect their life. Because some patients do not know the condition very well, but for various reasons, they are not willing to communicate with the medical staff, but at the same time, they want to know about the treatment-related information, inner contradictions, and anxiety. At present, chemotherapy drugs and some auxiliary drugs for breast cancer are expensive, and patients often need multiple courses of treatment, which will increase the economic burden of families. At this time, the increase in medical expenses makes some patients unable to afford, and anxiety and depression appear.

\subsection{Relationship between Sleep Status and Cancer-Related} Fatigue. The results showed that according to the cut-off value of sleep disorder of no less than 6 points, $60 \%$ of the subjects had sleep disorders. Insomnia is the most common sleep disorder in chemotherapy patients. Studies have shown that the incidence of insomnia in cancer patients during hospitalization is $67 \%$. The possible reasons for this result are patients' ignorance of cancer-related knowledge, and fear and anxiety about cancer itself will affect their sleep state. In the process of chemotherapy for breast cancer patients, the discomfort caused by chemotherapy and the worry about the prognosis of the disease will make the sleep quality of patients worse. There is a real psychological gap in the life of breast cancer patients before and after their illness. The patients worried about high medical costs, the breakdown of family relations, future work, not adapting to the hospital ward environment, dependence on sleeping pills, or withdrawal symptoms.

\section{Research Object and Operation Process}

3.1. Research Design. First of all, we need to determine the purpose of the study and the content to be studied, reasonably select the research object for the experiment according to the random selection method, and then test the blood sample of the research object. Then, a reasonable football program was developed; the subjects were trained for 6 months while the control group did not need any relevant training; only the conventional nursing mode was used. During the research process, we will test and record the indicators of patients in the intervention group and the control group for many times, and the test results were compared within and outside the group. Finally, the data were collected and sorted out to determine that the ability of patients with breast cancer treated by nano-chemotherapy after breast cancer surgery to resist fatigue was more significant due to the participation of football.

3.2. Research Object and Inclusion Criteria. The subjects of this study were breast cancer patients with fatigue undergoing chemotherapy in the breast surgery department of a hospital in Liaoning Province from March 2018 to 2018. They were aged from 30 to 45 years old; the total score of the cancer fatigue scale, i.e., fatigue, was used; patients who had the conditions to practice football at home voluntarily joined the study after the informed consent form was signed by patients and their families.

\section{Inclusion Criteria:}

(1) Female patients who were with breast cancer confirmed by clinical pathology and who underwent a modified radical mastectomy, clinical stage II, pathological type of invasive ductal carcinoma, and chemotherapy regimen of TC (docetaxel, cyclophosphamide) were included

(2) 24 hours after chemotherapy, the way of aerobic exercise was walking

(3) No metastasis was found

(4) Age was 30-55 years old

(5) If the total score of the cancer fatigue scale is greater than 0 , there is fatigue

(6) After informed consent and signed informed consent, they voluntarily joined the study

Exclusion Criteria:

(7) Patients with cancer or other body diseases such as cardiovascular disease, diabetes, hypertension, etc., were excluded

(8) Patients who were diagnosed with breast cancer without chemotherapy or preoperative chemotherapy or only radiotherapy and chemoradiotherapy, targeted therapy, and other comprehensive treatment were excluded and if metastases were found

(9) Patients who had past or present mental illness or disturbance of consciousness were also excluded

(10) Regular exercise habits were observed after the operation (moderate-intensity aerobic exercise $>3$ times a week, exercise time $>90$ minutes)

\subsection{Ethical Principles}

(1) Informed consent [13]: on the one hand, it explains the purpose and significance of the research to the 
data acquisition unit and obtains its consent and permission; on the other hand, it introduces the research purpose, content, process, and the possible benefits of participating in the research. According to the principle of voluntariness, this study is only an observational study, and the results are only used for the analysis of the experiment, not involving the interests of the subjects. At the same time, all subjects were required to sign the informed consent form entitled "the effect of aerobic exercise at different times on cancer-induced fatigue of breast cancer patients undergoing chemotherapy"

(2) Privacy and confidentiality: to protect the privacy of patients and inform the included subjects that the data will be strictly confidential and only used for this study

(3) Fairness: after the end of the study, if the results show that the effect of football intervention is good, we will contact the patients in the control group, inform them of the research results, and recommend to help the patients in the control group to carry out rehabilitation training

3.4. Assessment Tools and Contents. This paper takes the form of a questionnaire for evaluation and statistics:

(1) General information: the questionnaire was used to investigate age, education, education level, occupation, economic income, medical payment method, operation method, chemotherapy scheme, regular exercise habit after the operation, etc. The general information of this study is gender, residence, religious belief, nationality, marital status, fertility, family per capita monthly income, family relationship, personality type, housework, appetite, payment method, etc. Characteristics of clinical diseases: clinical stage, pathological type, operation type, chemotherapy scheme, complications, chemotherapy cycle, anemia, nausea and vomiting, pain, dry mouth, bitter mouth or oral ulcer, and other side effects.

(2) Cancer-related fatigue: the revised fatigue scale (RPFS) was used to measure the fatigue. The scale was mainly composed of 22 items in four parts, and the behavioral severity, emotional aspect, sensory aspect, and cognitive emotion of fatigue were tested, respectively. The scale is widely used at home and abroad and has good reliability and validity. In this study, the expert validity (CVI) of the scale is 0.87 ; the higher the score of the scale, the more serious the fatigue. Experts believe that the scale score less than or equal to 3 represents mild fatigue, that between 3 and 6 represents moderate fatigue, and that higher than 6 represents severe fatigue.

(3) Quality of life [14]: using a cancer treatment function evaluation system, breast cancer patient-specific scale measurement was used. There were five parts in the scale, including physical status (6 items), social and family status ( 6 items), personal emotional status (6 items), physical function status (6 items), and breast cancer-specific module (6 items). The 30 items of FACT-B are all classified into five levels: none (0), some (1), some (2), equivalent (3), and extraordinary (4). The higher the total score of the final statistical scale, the better the quality of life of patients. FACT$\mathrm{B}$ has been proved to be a breast cancer patientspecific scale with good reliability and validity. This is also confirmed in the preliminary experiment of this study.

(4) Anxiety and depression questionnaire: the scale is a general hospital anxiety and depression scale and is a common tool for the evaluation and management of emotional disorders in general hospitals. The hospital anxiety and depression scale (has) includes 14 items, which are composed of anxiety and depression. Two subscales, including seven items, are used to evaluate anxiety and depression, and the other seven items are used to evaluate the state. The scores of the two subscales are from 0 to 21 points, and the lowest score is 0 . According to the criteria of the author's evaluation, the key differences of anxiety and depression subscale were asymptomatic $0-7$, suspected anxiety and depression 8-10, and certain anxiety and depression 11-21. In addition, the two subscales were set with 8 points; that is, the patients suspected or confirmed anxiety and depression were positive for anxiety and depression. The reliability and validity of the hospital anxiety and depression scale were tested for the first time in China. The results showed that the Cronbach coefficient of the internal consistency of the anxiety scale was 0.92 , and that of the depression scale was 0.84 .

(5) Sleep status table: in this study, insomnia was evaluated. Sleep was the main content of the Athens Insomnia Scale. The subjective feeling of sleep and sleep state were evaluated. It includes eight items: induction of sleep within a month, waking up at night, getting up in the morning, total sleep time, sleep quality, mental state, physical function, and daytime sleepiness. Moreover, each item was scored by Likert 4 points ( $0-3$ points), and the total score of each item was accumulated. The critical points were as follows: the total score of no sleep disorder was no more than 3 points, the total score of possible sleep disorder was between 4 and 5 points, and the total score of sleep disorder was more than 6 points. The Chinese version of the scale has good reliability and validity with a Cronbach coefficient of 0.825 .

3.5. Football Intervention Methods. According to the specific situation of each patient (different time and different recovery levels after the operation), the action of football practice was guided. Accompanied by professional football teachers, researchers, and chief doctors, according to the specific physical conditions of breast cancer patients, the rehabilitation football sport plan suitable for patients with 
breast cancer after nano-chemotherapy technology in the initial, middle, and late stages is formulated, and the football sport teaching frequency is coordinated. The subjects and their families were trained on how to measure heart rate during exercise and the degree of mastery was investigated. Since the chemotherapy regimen is TC, the patient can be discharged without discomfort on the second or third day after chemotherapy, so the family members of patients are encouraged to accompany and supervise them to complete walking exercise after discharge. Patients were informed that they should stop the exercise immediately in case of nausea, vomiting, and irregular heart rate during exercise.

(1) Centralized intervention: during the period of nanochemotherapy after breast cancer surgery, the patients were accompanied by the chief physician and professional teachers of the breast department. Football practice was conducted three times a week, once every 2 days, for 1-2 hours each time. Until the end of the study, rehabilitation football CD was presented when discharged from the hospital.

(2) Decentralized intervention: during the intermission of chemotherapy, the patients should practice football by themselves according to the football education video at home, preferably accompanied by family members, three times a week, once every 2 days, 1 to 2 hours each time, until the patient's next chemotherapy admission. The patients were asked to record the frequency of football practice in the register. The researchers regularly followed up the patients by telephone and video to monitor the exercises.

\section{Results and Discussion}

4.1. Questionnaire for General Information of Patients. As shown in Table 1, we have made a simple table from the patient's living conditions, marital status, education, and patient's appetite, which can intuitively see the basic situation of the volunteers participating in the study. There was no significant difference between the two groups.

\subsection{Effect of Football Intervention on Cancer-Related Fatigue.} We analyzed the data based on the interval time of each chemotherapy. The objective is to explore the effect of football intervention on the fatigue of patients with chemotherapy. The results showed that after the intervention, the scores of total fatigue, physical fatigue, emotional fatigue, and cognitive fatigue in the intervention group were lower than those in the control group.

As shown in Figure 1, sample 1 represents the first sampling, sample 2 represents the second sampling, sample 3 represents the third sampling, and sample 4 represents the fourth sampling. The four-sample comparison shows that the score of physical fatigue has a significant decline, while the emotional fatigue and cognitive fatigue are obvious enough, but the data score still has a decline.
TABLE 1: Questionnaire of general information of patients.

\begin{tabular}{lccc}
\hline Project & $\begin{array}{c}\text { Experience } \\
\text { group }\end{array}$ & $\begin{array}{c}\text { Control } \\
\text { group }\end{array}$ & $P$ \\
\hline Place of residence & 13 & 15 & 0.789 \\
$\quad$ Countryside & 17 & 15 & \\
Town & & & 0.996 \\
\hline Marital status & 27 & 28 & \\
Married/ & & & \\
Cohabitation & 3 & 2 & \\
Divorced/ & & & 0.968 \\
Unmarried & 4 & 4 & \\
\hline Appetite & 5 & 7 & \\
Very good & 11 & 9 & \\
Good & 4 & 5 & \\
Commonly & 6 & 5 & \\
Poor & & & 0.798 \\
$\quad$ Very poor & 3 & 4 & \\
\hline Degree of education & 6 & 8 & \\
Primary school & 14 & 11 & \\
Junior middle school & 7 & 7 & \\
High school/College & & & \\
$\quad$ University or above & &
\end{tabular}

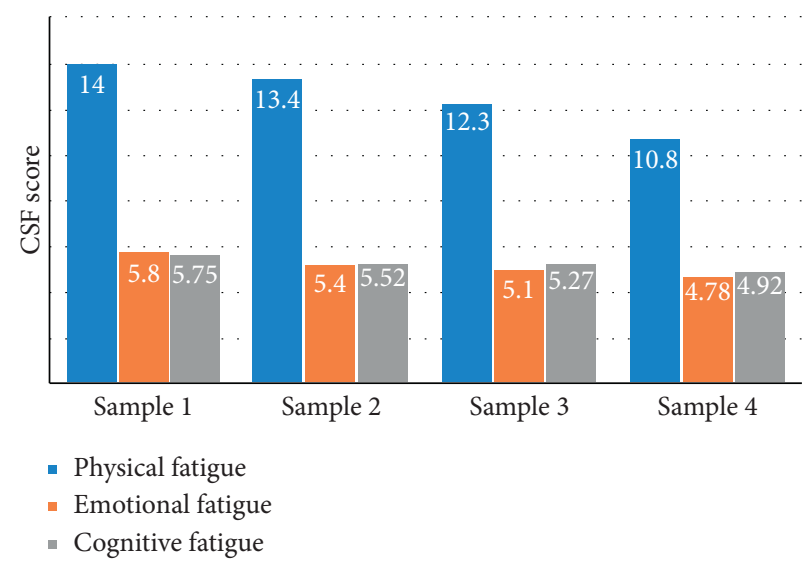

FigURE 1: Changes of CFS score in the intervention group after football intervention.

4.3. Influence of Football Intervention on the Quality of Life. After the intervention, the quality of life scores of the intervention group and the control group at the third, sixth, and ninth chemotherapies are shown in Table 2.

As shown in Table 2, the results showed that after the intervention, in addition to the score of functional status, the scores of the overall quality of life, physiological status, family status, emotional status, and breast cancer additional attention of the intervention group were generally higher than those of the control group at each time point. The score of the functional status of the intervention group was lower than that of the control group at the third chemotherapy and higher than that of the control group at the sixth chemotherapy.

After the intervention, the scores of the quality of life between the two groups were compared.

As shown in Figure 2, in the comparison of the scores of the quality of life between the two groups after the 
TABLE 2: Quality of life scores of the intervention group and control group in the third, sixth, and ninth chemotherapies.

\begin{tabular}{|c|c|c|c|c|c|c|}
\hline \multirow[b]{2}{*}{ Project } & \multicolumn{3}{|c|}{ Experience group } & \multicolumn{3}{|c|}{ Control group } \\
\hline & $\begin{array}{c}\text { The third } \\
\text { chemotherapy }\end{array}$ & $\begin{array}{c}\text { The sixth } \\
\text { chemotherapy }\end{array}$ & $\begin{array}{c}\text { The ninth } \\
\text { chemotherapy }\end{array}$ & $\begin{array}{c}\text { The third } \\
\text { chemotherapy }\end{array}$ & $\begin{array}{c}\text { The sixth } \\
\text { chemotherapy }\end{array}$ & $\begin{array}{l}\text { The ninth } \\
\text { chemotherapy }\end{array}$ \\
\hline Physiological status & 15.1 & 15.8 & 17.5 & 16.3 & 14 & 13.6 \\
\hline Family status & 20.23 & 20.05 & 20.0 & 19.5 & 19.2 & 18.9 \\
\hline Emotional state & 15.80 & 15.81 & 16.89 & 15.34 & 14.58 & 15.34 \\
\hline Functional status & 12.24 & 12.31 & 14.13 & 13.27 & 12.85 & 13.81 \\
\hline Additional attention to breast cancer & 22.59 & 23.74 & 25.12 & 21.54 & 20.69 & 20.64 \\
\hline Overall quality of life & 85.96 & 87.71 & 93.64 & 85.95 & 81.32 & 82.29 \\
\hline
\end{tabular}

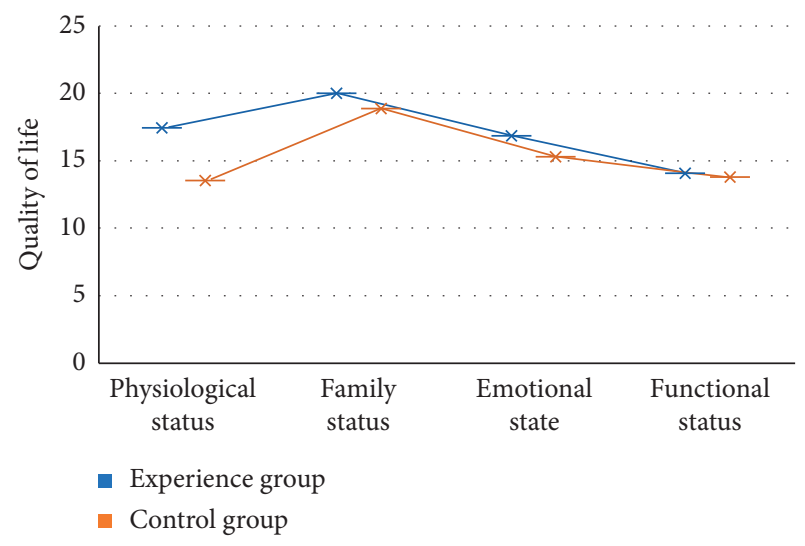

Figure 2: Comparison of quality of life scores between the two groups after the intervention.

intervention, we selected the data of the 9th chemotherapy to compare and analyze the patients in the intervention group and the control group, respectively, from the physiological status, family status, emotional status, and functional status. As shown in the figure, we can more intuitively see that the quality of life in the intervention group is significantly better than that in the control group.

4.4. Effect of Football Intervention on Sleep Quality. As shown in Figure 3, sample 1 represents the second chemotherapy, sample 2 represents the fourth chemotherapy, sample 3 represents the sixth chemotherapy, sample 4 represents the eighth chemotherapy, and sample 5 represents the tenth chemotherapy. Through the sleep quality records of the ten chemotherapy time cycles, the sleep quality of patients after the intervention is getting better and better, especially during the second to the fourth chemotherapy where it has an obvious effect. During this period, the sleep quality was improved rapidly, and it entered a stable state after the sixth chemotherapy. Therefore, adherence to football training can improve the quality of sleep and also can let patients have a full rest to achieve the effect of antifatigue.

4.5. Influence of Football Intervention on Anxiety and Depression. As shown in Figure 4, sample 1 represents the first chemotherapy, sample 2 represents the second chemotherapy, and sample 3 represents the third chemotherapy. For the influence of football intervention on the anxiety

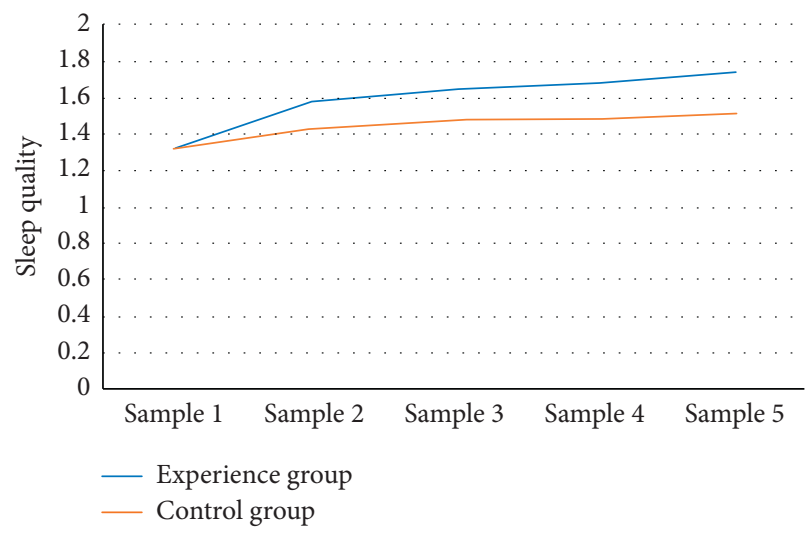

FIGURE 3: Effect of football intervention on sleep quality.

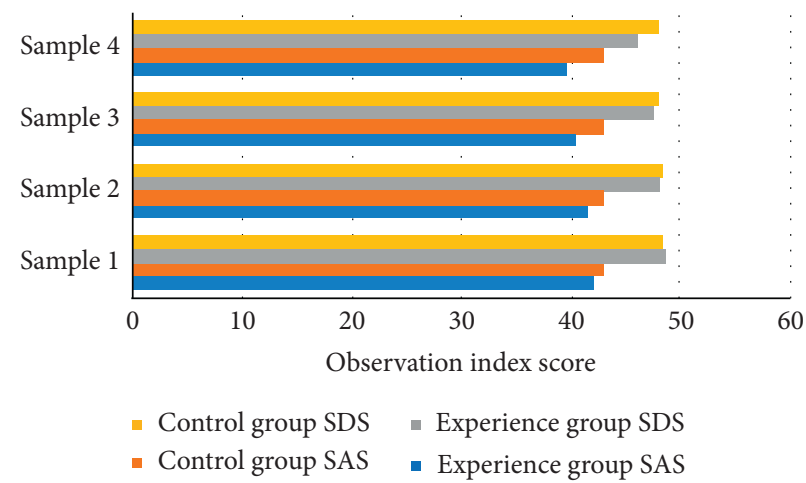

Figure 4: Score of anxiety and depression of football intervention.

and depression of chemotherapy patients, we compared the data of the intervention group and the control group, respectively. It can be seen that whether it is depression or anxiety, the score of the intervention group is lower than that of the control group. It can be concluded that football can significantly improve the anxiety and depression of patients with breast cancer after nano-chemotherapy.

\section{Conclusion}

Breast cancer is one of the main malignant tumors endangering women's health. Since the end of the century, the incidence of breast cancer has been in the first place in the world, and it is increasing at an annual rate. There are about 
ten thousand women suffering from breast cancer every year in the world, and ten thousand women die of breast cancer. Cancer-related fatigue is the most common and very difficult to control the subjective and continuous fatigue feeling of patients after breast cancer surgery, which seriously affects the rehabilitation effect and quality of life of patients. This study explored the effect of football intervention on cancer-related fatigue, quality of life, sleep, anxiety, depression, and other conditions of breast cancer patients, in order to explore a better exercise program that can make patients actively carry out rehabilitation exercise and have good rehabilitation effect and provide theoretical basis and empirical basis for the research on cancer-related fatigue and quality of life of patients after breast cancer surgery. In this paper, we use the sublease intervention comparative experiment. We divided the volunteers into two groups: the intervention group and the control group. The control group was given routine treatment and nursing in the breast department, while the intervention group was given football practice from the first chemotherapy on the basis of routine treatment and nursing, and the patients were required to record their sports. With the participation of professional football teachers, doctors, and nurses, different guidance was given according to the different conditions of each patient. The patients were admitted to the hospital from the first chemotherapy. The intervention time of each patient was about 6 months. After the observation and comparison of the intervention group and the control group, we concluded that football can reduce physical fatigue, emotional fatigue, and cognitive fatigue in different degrees; compared with the control group, it greatly improves the physiological status, family status, emotional status, and functional status. Football training can improve the quality of sleep, improve the anxiety and depression of patients, and also can let patients have a full rest to achieve the effect of resist fatigue. Therefore, football can be used as a clinical intervention method to deal with breast cancer disease and disease treatment-related side effects, but collective intervention is very important, which helps to improve the compliance of patients. Therefore, clinical workers can observe the long-term effect of football on breast cancer patients.

\section{Data Availability}

No data were used to support this study.

\section{Conflicts of Interest}

The authors declare that there are no conflicts of interest regarding this submission.

\section{Acknowledgments}

This work was supported by 2018 Hunan Provincial Department of Education General Project Fund (Project no. 18C044), the Teaching Research Project of , and Philosophy and Social Science Fund of Hunan Province (No. 19YBA124).

\section{References}

[1] J. D. Shull, K. L. Dennison, A. C. Chack, and A. Trentham-Dietz, "Rat models of $17 \beta$-estradiol-induced mammary cancer reveal novel insights into breast cancer etiology and prevention," Physiological Genomics, vol. 50, no. 3, pp. 215-234, 2018.

[2] T. W. Hoenemeyer, T. J. Kaptchuk, T. S. Mehta, and K. R. Fontaine, "Open-label placebo treatment for cancerrelated fatigue: a randomized-controlled clinical trial," Scientific Reports, vol. 8, no. 1, p. 2784, 2018.

[3] Z. Veqar and M. E. Hussain, "Psychometric analysis of Epworth sleepiness scale and its correlation with Pittsburgh sleep quality index in poor sleepers among Indian University students," International Journal of Adolescent Medicine and Health, vol. 31, no. 2, pp. 30-36, 2018.

[4] K. Cosansu, C. M. Ureyen, and S. Y1lmaz, "Effect of novel oral anticoagulants on hospital anxiety and depression scale scores," Herz, vol. 44, no. 8, pp. 743-749, 2019.

[5] M. Babincová, H. Vrbovská, P. Sourivong, P. Babinec, and Ś Durd e, "Application of albumin-embedded magnetic nanoheaters for release of etoposide in integrated chemotherapy and hyperthermia of u87-mg glioma cells," Anticancer Research, vol. 38, no. 5, pp. 2683-2690, 2018.

[6] F. L. Camati, O. ., F. G. Assunção, L. S. Kely, B. Daniel, B. Romulo, and L. S. A. Eduardo, "Caffeine increases both total work performed above critical power and peripheral fatigue during a 4-km cycling time trial," Journal of Applied Physiology, vol. 124, no. 6, pp. 1491-1501, 2018.

[7] C. Audrey, R. Raquel, B. Andrea et al., "Effects of glutamine and alanine supplementation on central fatigue markers in rats submitted to resistance training," Nutrients, vol. 10, no. 2, p. 119, 2018.

[8] D. N. Jarvis and R. E. Abergel, "What do we know about how acute physical fatigue affects movement in dancers?: a systematic review of the literature," Medical Problems of Performing Artists, vol. 34, no. 3, pp. 161-168, 2019.

[9] V. Zsolnay, M. Fill, and D. Gillespie, "Sarcoplasmic reticulum $\mathrm{Ca}^{2+}$ release uses a cascading network of intra-SR and channel countercurrents," Biophysical Journal, vol. 114, no. 2, pp. 462-473, 2018.

[10] S. Ling and L. Hui, "Evaluation of the complexity of indoor air in hospital wards based on PM2.5, real-time PCR, adenosine triphosphate bioluminescence assay, microbial culture and mass spectrometry," BMC Infectious Diseases, vol. 19, no. 1, p. 646, 2019.

[11] L. Kai, W. Yong, Z. Anji, L. Baixue, and J. Li, "Mir-379 inhibits cell proliferation, invasion, and migration of vascular smooth muscle cells by targeting insulin-like factor-1," Yons Medical Journal, vol. 58, no. 1, pp. 234-240, 2017.

[12] P. F. Parvin and F. Parvin, "Aromatase inhibitors as adjuvant therapy in postmenopausal women with early-stage hormone receptor positive breast cancer," Current Opinion in Obstetrics and Gynecology, vol. 30, no. 1, pp. 51-54, 2018.

[13] J. D. Rendtorff, "Update of european bioethics: basic ethical principles in european bioethics and biolaw," Bioethics Update, vol. 1, no. 2, pp. 113-129, 2015.

[14] M.-H. Shen, L.-P. Chen, T.-F. Ho et al., "Validation of the taiwan Chinese version of the EORTC QLQ-CR29 to assess quality of life in colorectal cancer patients," BMC Cancer, vol. 18, no. 1, p. 353, 2018. 